\title{
SOBRE LOS NOMBRES DE LAS UNIDADES FAMILIARES INDÍGENAS EN LA HISPANIA ANTIGUA (2. ${ }^{\text {P }}$ PARTE)*
}

\section{ON THE NAMES OF THE INDIGENOUS FAMILY UNITS IN ANCIENT HISPANIA ( $2^{\text {nd }}$ PART)}

\author{
EUGENIO R. LujÁN \\ Universidad Complutense de Madrid \\ erlujan@ucm.es \\ ORCID: 0000-0002-6769-3791
}

DOI: $10.1387 /$ veleia.18082

\begin{abstract}
Resumen: Se estudia en este trabajo la relación entre los nombres de unidades familiares indígenas en genitivo plural y los nombres indígenas que también puede referirse a ellas, atendiendo a diferentes aspectos. Tras analizar en la primera parte del artículo los procedimientos de derivación empleados para formar los nombres de esas unidades, en esta segunda parte se lleva a cabo una revisión de las menciones de parentesco en relación con los nombres de unidades familiares, con especial atención al tercer bronce de Botorrita. Los resultados refuerzan la idea de que no se trataba de grandes clanes, sino de grupos más limitados en número y extensión temporal, lo que se puede poner en relación con la información epigráfica sobre los procesos por los cuales podían ir surgiendo, dentro de una unidad familiar previamente existente, nuevas unidades con un nombre diferente, que ya fueron tratados en la primera parte.

Palabras clave: unidades familiares indígenas, fórmulas onomásticas, epigrafía latina, celtibérico, Hispania antigua.

Abstract: This paper aims at offering a revision of the relationship between the names of indigenous family units in genitive plural attested on the inscriptions of Ancient Hispania and the indigenous names found in onomastic formulae that can also be used to refer to these units. As a continuation of a Veleia 2016 study on the various suffixes used for deriving these names, we present in this paper the results of a comprehensive reexamination of the documents including kinship terms together with names of family units, and paying special attention to the third Botorrita bronze. The data support the idea that these family units were not big tribes or clans, but smaller groups with a limited duration in time. This can be connected to the information provided by a number of inscriptions concerning the emergence of new family units provided with a new name inside previously existing units that we dealt with in the first part of the paper.

Keywords: indigenous family units, naming formulae, Latin inscriptions, Celtiberian, Ancient Hispania.
\end{abstract}

Recibido: 18-06-2014

Informado: 29-04-2015

Definitivo: 29-11-2016

Continuación de la primera parte, publicada en el número anterior de la revista Veleia. 


\title{
3. UNIDADES FAMILIARES Y PARENTESCO ${ }^{1}$
}

Ya hemos mencionado en la primera parte de este trabajo, al hilo del comentario de algunas inscripciones, la cuestión de las relaciones de parentesco a propósito de la pertenencia a una unidad familiar. Convendrá, por tanto, replantearse dos temas debatidos en la investigación sobre las unidades familiares y estrechamente conectados entre sí: cuál era el principio que regía la pertenencia a una de estas unidades familiares y cuál era el modo de atribución de los nombres de las unidades. Para ello ofrecemos a continuación de forma exhaustiva la documentación con que contamos hoy en día sobre parentesco y pertenencia a una misma o a distinta unidad familiar.

Desde un punto de vista social, resulta interesante constatar que tenemos inscripciones en las que marido y mujer pertenecen a dos estructuras diferentes. El ejemplo más claro a este respecto lo constituye la siguiente inscripción ${ }^{2}$ :

\section{CIL II $6294=$ HEpOL 103 (Almadrones GU):}
a) Atta $\cdot$ Abbloiocum / Rectugelni $\cdot f($ ilia $) \cdot L(u c i) \cdot u x o r / h(i c) \cdot s($ ita $) \cdot e(s t) \cdot s($ it $) \cdot t($ ibi $)$. t(erra) $\cdot$ l(euis)
b) Luciu[s] / Nissic(um) / Accuti / f(ilius $) \cdot h($ ic $) \cdot s($ itus $) \cdot e(s t) \cdot s($ it $) \cdot t($ ibi $) \cdot t($ erra $) \cdot l($ euis $)$

Como se comprueba, la mujer pertenece a la unidad de los Abboioci, mientras que el marido pertenece a la de los Nissici. Una situación semejante nos encontramos en una inscripción de Medinaceli SO:

\begin{abstract}
CIL II 5789 = HEpOL 12094: Valerius / Bedaciq(um) / Candidus / h(ic) s(itus) e(st) / [ual] e(?) // Valeria / Venniq(um) / Successa / Candidi u(xor) / u(iua) s(ibi) et marito / f(aciendum) c(urauit) // T(itus) Licinius / Quir(ina) / Titulus / Cornutanulus / h(ic) s(itus) e(st) Suc(cessa) s(o)r(or) / d(e) s(uo) f(aciendum) c(urauit)
\end{abstract}

\footnotetext{
${ }^{1}$ Este trabajo forma parte del proyecto de investigación «Estudios de morfología nominal: lenguas paleohispánicas e indoeuropeas antiguas» (FFI2012-36069-C03-02), financiado por el Ministerio de Economía y Competitividad y ha sido realizado dentro del Grupo de Investigación consolidado de la Universidad Complutense de Madrid "Textos epigráficos antiguos de la península Ibérica». Estoy muy agradecido al Prof. Javier de Hoz por sus comentarios y sugerencias sobre versiones previas. También quiero agradecer las referencias bibliográficas y los comentarios del Consejo de Redacción de la revista Veleia, así como las observaciones y sugerencias de dos informantes anónimos. Obviamente, la responsabilidad de cualquier error u omisión es únicamente mía.

Para los corpora epigráficos las abreviaturas utilizadas son las de la revista Hispania Epigraphica. No podemos reflejar de forma exhaustiva la bibliografía existente para cada inscripción citada en el trabajo, por lo que nos li-
}

mitamos a dar una referencia que permita identificarla (normalmente el CIL II, un corpus o su primera aparición en las revistas Hispania Epigraphica, Année Épigraphique o similar), seguida de la equivalencia en la base de datos Hispania Epigraphica On-Line (http://eda-bea. es/), donde se pueden encontrar fácilmente las referencias a la bibliografía anterior.

${ }^{2}$ Los ejemplos conocidos hasta el momento de publicación del libro, como este mismo y el siguiente, ya son mencionados y analizados por González 1986, 101-106. No incluimos la inscripción de Tarragona (CIL II 4233) a la flaminic(ae) p(roninciae) H(ispaniae) $C$ (iterioris), que es ex gente Cantabro(rum), hecha por su marido, que es ex gente Vaccaeor(um) porque, aunque se utilice el término gens, no parece que se trate de unidades familiares indígenas del mismo orden que las que estamos analizando aquí. Sobre el significado de gens véase González 1986, 77-110. 
Aunque no se menciona explícitamente la relación de parentesco existente entre los difuntos lo más probable es que también los individuos mencionados en una inscripción de Ávila fueran marido y mujer ${ }^{3}$ :

ERAv $40=$ HEp OL 6352:

a) Dobitlerus . Claburolniq(um) Equalesi f(ilius) / [s(it) t(ibi)] t(erra) l(euis)

b) Aren/a $\cdot$ Melntoulieq(um) / Aelci / f(ilia) s(it) $\cdot t$ (ibi) $\cdot$ t(erra) / l(euis)

Asimismo, expresado mediante fórmula con duo nomina y nombre único, tenemos el ejemplo siguiente (Ramírez 1999, 461-463):

ERS 112 (Tordesalas SO): M(arco) - Laturico $\cdot L($ ucii) $\cdot$ f(ilio) / Alionicae - matri / et eius filiae $[--]$

Sin embargo, en otras ocasiones se menciona de forma conjunta a marido y mujer como pertenecientes a una misma unidad familiar (González 1986, 102):

ERA 41 = HEpOL 14497 (Beleño O): Peregrius / et Pompe(ia) Ar/nunimo/ru[m] / fili(a)e su(a)e pien/ti(s)sim(a)e Supel[ri(a)e]

CIL II 3121 = HEpOL 8941 (Uclés CU): Bru(tus) Satullus / Druttia Festa / Lougeidocum / h(ic) s(iti) s(unt) / Fuscus patri et sibi matri

A los ejemplos de González se puede añadir ahora dos más en los que se menciona explícitamente la unidad familiar de ambos cónyuges por separado (Ramírez 1999, 239; González y Ramírez 2011, 257):

HEp.7.343= HEpOL 116 (Gualda GU):

a) Atta / Turoco(m) / G(ai) u/xor / h(ic)s(ita) e(st)s(it) t(ibi) t(erra) l(euis)

b) Gaius / Turoco(m) / Pacati / f(ilius) $\cdot u i u(u) s \cdot / s(i b i) \cdot m(o n u m e n t u m) \cdot f($ aciendum) . c(urauit).

Barbas, Gamo y Gimeno 2011, n.o 1:

a) Luc[-]a / Obidoq(um) / Acconis / (-vacat-) ux (or) / h(ic) $\cdot s($ ita $) \cdot e($ st $) \cdot s($ it $) \cdot t($ ibi $) \cdot t($ erra $)$. l(euis)

b) Acco O/bidoq(um) / Val(erii) f(ilius) / [ann(orum)] XXXX/ h(ic) $\cdot s($ itus $) \cdot e(s t) \cdot s($ it $) \cdot t($ ibi $)$. t(erra) $\cdot$ l(euis)

3 También puede que sea un caso similar el que atestigua una una inscripción de Sepúlveda SG (HEp.3.334 = HEpOL 15359, cf. Ramírez 1999, 355-357), pero su mala conservación no permite reconstruir las fórmulas onomásticas ni tampoco la relación de parentesco que se menciona en la línea 4, pues se ha restituido tanto [parent]ibus como [fratr]ibus, si bien la primera posibi- lidad es la más probable, habida cuenta de que los genitivos de plural [---]rioq(um) y [---]+aniq(um) que aparecen en las líneas 2 y 3 dejan ver que se trata de unidades familiares diferentes, lo que, como estamos viendo, es posible cuando se trata de marido y mujer, pero no es esperable si se tratara de hermanos del dedicante, como veremos más abajo. 
Aunque no se menciona explícitamente la relación marital, es la interpretación más probable también para la siguiente inscripción:

CIL II $3110=$ HEpOL 8940 (Saelices CU): Iulius Hilario / et / Montana Con/tucianco(m) / h(ic) s(iti) s(unt) / Iulius Hilario

Tampoco podemos estar seguros, pero cabe considerar esa misma posibilidad, la de que se trate de marido y mujer, para los individuos mencionados en las siguientes inscripciones ${ }^{4}$ :

\author{
ERAv $164=$ HEpOL 25647 (Candeleda AV): Eburein/ius $\cdot$ Curunldi $\cdot$ Caraleciq (um) \\ ERAv $134=$ HEpOL 26015 (Narros del Puerto AV): [A]tta $\cdot$ Lugua / Caraecicu/m Eburen/i. \\ uxor
}

Conviene introducir en este punto la discusión sobre launi, si bien se trata de una palabra celtibérica de cuyo significado no podemos estar completamente seguros. Aparece en el tercer bronce de Botorrita (MLH IV, K.1.3) y quizá también en un grafito sobre cerámica campaniense de Contrebia Belaisca ${ }^{5}$. J. Untermann ${ }^{6}$ propuso, por sus combinaciones dentro de la relación de personas mencionada en el bronce, que fuera un apelativo que designara un tipo de relación de parentesco. launi siempre va precedido de una secuencia de nombre propio masculino con nombre de familia en genitivo y, a su vez, a launi puede seguirle o no otro nombre de familia en genitivo. El hecho de que cuando le sigue un nombre de familia este sea distinto del que le precede hace pensar, siguiendo a Untermann, que no puede tratarse de una persona de la misma descendencia, pues se esperaría que perteneciera a la misma unidad familiar. Por otro lado, el final en $-i$ invita a pensar que nos encontramos ante un femenino en $-\bar{\imath}\left(<^{*}-i h_{2}\right)^{7}$. La interpretación de launi como la palabra para "esposa» en celtibérico cuadra bien, por tanto, con lo que llevamos visto hasta ahora en las inscripciones latinas. En el tercer bronce de Botorrita tendríamos atestiguados así los dos tipos de casos que hemos visto en la epigrafía latina:

a) marido y mujer pertenecen a la misma unidad familiar y, por tanto, no resulta necesario repetir la mención:

elu : uiriaskum : launiku[e] (II.5)

terkinos : atokum : launikue (III.40)

d) marido y mujer pertenecen a distintas unidades familiares y, por tanto, hay que indicar también la de la mujer:

elkuanos : kunikum : launikue : uiriaskum (II.27-28)

turaios : litanokum : kurmilokum : launikue : uiriaskum (III.57-58)

4 La cronología que se puede dar a las dos inscripciones no contradice esta posibilidad; véanse los comentarios de Hernando en ERAv 134 y 164.

5 Cuya lectura es, según Untermann ( $M L H$ IV, K.1.4), ]: aultu launikue[, aunque Prósper 2007, 161166 ha propuesto leer ] auz : kelauniku[.
6 En Beltrán, De Hoz y Untermann 1996, 119-120.

7 Como indica Jordán 2004, 83 y 125, quien también revisa el significado de la palabra en las págs. 171172 y da «esposa» entre interrogaciones. 
Más hipotética aún es la relación existente entre los individuos mencionados en secuencias del bronce como la siguiente ${ }^{8}$ :

tiriu : uiriaskum turtunazkue : kazarokum (III.31-32)

La utilización de la conjunción copulativa -kue uniendo las dos entradas indica sin duda que existe algún tipo de relación entre los dos individuos y turtunaz- parece un nombre femenino9 ${ }^{9}$, por lo que quizá se trate también de un matrimonio; en tal caso marido y mujer pertenecerían a unidades familiares diferentes.

Podemos mencionar también otros casos como los siguientes:

abu++ akuiakue : araiokum (I.42)

akuia : sekiloskue : tirilokum (II.11)

kalaitos koitinakue : uiri \{r\}askum (II.33-34)

elku : suolakue tirtanikum : uiriaskum : $\operatorname{mel}(---)$ (II.2-3)

toloku : koitinakue : austunikum (III.5)

arkanta : mezukenoskue : abokum (III.11)

abaliu berikakue : suaikinokum (III.25)

bilir+ turtuntakue : telkaskum (III.38)

usama : abaloskue : karunikum (III.47)

ultinos : amakue uiriaskum (III.55)

En estas secuencias, a pesar de que los finales en - $a$ en uno de los nombres apuntan a que se trata de femeninos ${ }^{10}$ y la conjunción -kue muestra la relación existente entre ellos, resulta más arriesgado pensar en una relación familiar estrecha más allá de la pertenencia a la misma unidad familiar, puesto que -kue también se utiliza para unir dos nombres masculinos o una secuencia de varios nombres cuando los individuos pertenecen a la misma unidad familiar, como en:

or++bilos : likinoskue abo++kum (I.40-41)

alu : aiukue : araiokum (I.43)

misuku : retukenos : tirtanos munikakue uiriaskum (I.52-53)

ka<l>aitos : litukue : abokum (I.57)

mezukenos : elazunos tirtukue : ailokiskum (II.21-22)

Si pasamos ahora a ver qué sucede con los hijos, parece claro que estos pertenecen a la misma unidad familiar que su padre, tal y como muestra el ejemplo siguiente, ya mencionado por González 1986, 103:

HEp.4.645 = HEpOL 15605 (Ventosilla y Tejadilla SE): P(ublio) Abianiclum an(norum) LX / (A)emi(lius) Abi(anicum) / Pro(culus) Abi(anicum) / patri

8 En el caso de sanion : baatokum niskekue : babokum (I.35-36), que presentaría una estructura similar, de entrada la lectura de niskekue no es segura y podría ser también niskere (Beltrán, De Hoz y Untermann 1996, 47), con lo cual serían dos nombres sin relación entre sí. Y, aun cuando niskekue sea la secuencia correcta, la ausencia de paralelos onomásticos lleva a Untermann (ibidem, 150) a plantear la posibilidad de que se trate de un apelativo, quizá con el sentido de «sobrina».

\footnotetext{
9 Como indica Untermann en Beltrán, De Hoz y Untermann 1996, 162-163.

10 Véanse los comentarios de Untermann a los respectivos nombres en Beltrán, De Hoz y Untermann 1996, 121-166. En el caso de koitina se añade, además, el problema de si podría tratarse de un apelativo y no de un antropónimo (Untermann, ibidem, 142143).
} 
Consecuentemente con la pertenencia a la misma unidad de padre e hijo, los hermanos varones deberían pertenecer a la misma unidad familiar, como, de hecho, lo documentan las inscripciones de Armada LE, así como el primer bronce de Botorrita ${ }^{11}$ :

\section{CIL II $5720=$ HEpOL 12040 (Armada LE): M(onumentum) / Virono Taur $[i / n]$ o Doideri f(ilio) / uad(iniensi) annoru/m XL h(ic) s(itus) e(st) / Placidus au(u)/nculis pos(suit) \\ CIL II 5708 = HEPOL 12028 (Armada LE): M(onumentum) / Ablon/no Taurilno Doidleri . $f($ ilio $) \cdot$ uald(iniensi) annolrum XXX / h(ic) $\cdot$ s(itus) $\cdot e($ st $)$ / Placidus / a (u)unculis / po(suit)}

$M L H$ IV K.1.1: ueizu aiankum tauro y abulu aiankum tauro

La utilización de la palabra kentis «hijo» en el tercer bronce de Botorrita proporciona información adicional sobre la pertenencia de padre e hijo a la misma unidad. No parece que haya mayor problema en entender que se refieren a un individuo y su hijo las siguientes secuencias, en todas las cuales se menciona únicamente una unidad familiar, pues a ella pertenecen tanto el padre como el hijo ${ }^{12}$ :

rusku : uiriaskum : kentisku<e> (I.39)

turenta : kentiskue : ataiokum (II.25)

kinbira : kentiskue : turikum (III.4)

babos : kentiskue : uiriaskum (III.56)

En la primera de ellas (I.39) se ha añadido la mención «e hijo» tras dar el nombre personal del padre y su unidad familiar de pertenencia. Sin embargo, en las posteriores parece que se ha "estandarizado» la fórmula integrada por nombre personal + «e hijo» + nombre de familia en genitivo. Obsérvese que esta colocación mayoritaria de la secuencia «e hijo» entre el nombre del padre y el nombre de familia contrasta con la de la palabra launi "esposa» que vimos más arriba, que siempre aparece tras la mención de la unidad familiar del marido, pues cabe la posibilidad de que su unidad familiar sea distinta, algo que no puede ocurrir con el hijo.

La entrada más irregular de Botorrita III, como bien lo señala De $\mathrm{Hoz}^{13}$, es la que encontramos en III.23-24:

\section{retukeno : elkueikikum / kentisum : tuateroskue}

No sabemos la razón por la que de repente en esta secuencia se pasa al caso genitivo (retukeno y no nom. * retukenos, como sería lo esperable), cuando las entradas onomásticas del resto del bronce están en nominativo. Sin embargo, una vez asumida la incoherencia en cuanto al caso gra-

11 También, muy probablemente, la inscripción funeraria de Garray SO (HEp.11.518 = HEpOL 24595) dedicada a Abico Cas(a)r(i)co p(atri) et Ant(oniae) por sus hijos, un hermano y otra persona de la misma unidad familiar (nati et Caricu(s) C(asarico) frater et Titus Casarico(n) Saicli Calistratio), si bien, como se ve, la unidad familiar del hermano está abreviada.

12 En II.2.3 se lee: tirtanos kentiskue : loukaniko uiriaskum. Aunque aquí sí hay referencia a la unidad familiar, no hay por qué pensar que kentis va con tirtanos, sino que más bien parece que de él depende el genitivo que le sigue, loukaniko, es decir, «Tritano y el hijo de Lucanico, de los Viriascos». En la otra aparición de kentis no hay referencia a la unidad familiar, pero se inserta en un contexto similar, solo que acompaña a un nombre femenino (Flora): bolora : kentiskue : melmanzos (IV.3). Tampoco hay referencia a la unidad familiar en el único caso de uso de la abreviatura del nombre del hijo (ke) en III.45.

13 De Hoz en Beltrán, De Hoz y Untermann 1996, 96-97. Sin embargo, ninguna de las posibilidades que ofrece nos resulta convincente y, con Untermann (en Beltrán, De Hoz y Untermann 1996, 119) creemos que kentisum y tuateros son apelativos en genitivo, plural y singular, respectivamente. 
matical, la entrada resulta explicable, pues se trata simplemente de la mención en genitivo «y de los hijos e hija» (kentisum tuateroskue) que en este caso se coloca detrás de la unidad familiar a la que pertenece Re(c)tugenos.

Hay una aparición más del nombre "hija» en este bronce, concretamente en nominativo plural «hijas» (tuateres), que tampoco conlleva la referencia a una unidad familiar distinta:

\section{kekas : ko(---) / aureiaku / tuate+eskue : uiriaskum (II.39-40)}

Si padre e hija pueden pertenecer a la misma unidad familiar, entonces no sorprende que dos hermanas también puedan pertenecer a la misma, como lo muestran estas dos inscripciones (González 1986, 103): f(iliae)

CIL II 287* = HEpOL 191 (Escalonilla TO) ${ }^{14}$ : Optata et / Endeicte A/lbiganic(um) / Seru(ilii)

CIL II $6299=$ HEpOL 12615 (Aguilar de Campoo-Olleros de Pisuerga P):

a) $D$ (is) $M$ (anibus) Aiae Quemiae Boddi f(iliae) Celtigun an(norum) XXXI

b) $D$ (is) $M$ (anibus) Carauancae Boddi f(iliae) Celtigun an(norum) XXXV

c) Aia Origena Vironi f(ilia) monimentu $(m)$ faciendu $(m)$ curauit pientissimis filiabus

Precisamente en otro epitafio doble también de Monte Cildá, hecho por el dedicante en honor de su madre y de su tía materna, se atestigua la pertenencia de dos hermanas a la misma unidad familiar, expresada en este caso mediante el dativo femenino Caledige:

CIL II 6298 = HEPOL 12616 (Aguilar de Campoo-Olleros de Pisuerga P):

a) D(is) M(anibus) / Aninus / posui . I An/nae Caleldig(a)e matelrter(a)e · piale qu(a)e $\cdot$ uilcsit . aln.nis / LXXX

b) $D($ is) $M$ (anibus) / Aninus / filius / Douidelnae . Calledig(a)e / / matri · pliaenti(s)/sim(a)e . qulae uixsit annis / $X X \cdot V$

c) Aninus / indulgelntis(s)imils posuit

Sin embargo, que las hijas pueden no pertenecer a la misma unidad que el padre, queda claro en las siguientes inscripciones, la primera de ellas ya mencionada por González 1986, 103 :

EE IX $317=$ HEpOL $198($ Hontanar TO $):$ Licinia $\cdot$ Molenicu $(m) \cdot B($ outia $) \cdot$ Pater $/$ ni $\cdot$ Maganil $q($ um $) \cdot f($ ilia $) \cdot h($ ic $) \cdot s($ ita $) \cdot e($ st $) \cdot s($ it $) \cdot t($ ibi $) \cdot t($ erra $) \cdot l($ euis $)$

CIL II $5736=$ ERA 33 (Parres O): [Monument] um / p[ositum dib] us M/anibus Scorcia O/nnacau(m) Ammiae / Caelionigae ex / gente Pentioru(m) / anno(rum) XV / pater filiae / posuit / do(mino) no(stro) Pos(tumo) / IIII et Vict(orino) co(n)s(ulibus)

Quizá haya que buscar la explicación en la posibilidad de cambio de unidad familiar cuando la hija contraía matrimonio, en relación con los datos que venimos viendo sobre la pertenencia o no a la misma unidad familiar de marido y mujer.

14 Considerada falsa en el CIL II, si bien muchos estudiosos modernos la consideran auténtica, como se señala en la entrada de $H E p O L$. 
También resulta interesante constatar que la madre y la mujer de un mismo individuo pertenecen a unidades diferentes, como se muestra en una inscripción de Talavera de la Reina TO (González 1986, 103-104):

$A E$ 1969-70.252 = HEPOL 294:

a) Ammiale Pistirilcum Pistliri f(iliae) Aeg/andus et Amaenea / matri / f(aciendum) c(urauerunt)

b) Proculae Pentalniq(um) Boultif(iliae) Aeglandus / uxsori / f(aciendum) c(urauit)

Utilizando como evidencia también los nombres en -cus, se constata igualmente que un individuo y su madre pueden no pertenecer a la misma unidad familiar, como lo mostraría la siguiente inscripción:

CIL II 2866 = HEpOL 8694 (Lara de los Infantes BU): Coemeae Des/sicae Visad[---]qu/ini f(iliae) an(norum) LX C(aius) Vallerius Turancicus / matri

En efecto, si Dessicae y Turancicus son nombres que hacen referencia a unidades familiares, como los consideran González 1986, 136 y Ramírez 1999, 482, hijo y madre no pertenecerían a la misma.

En cambio, en la siguiente inscripción Iulius Barbarus y su madre Aemilia Acca sí que pertenecen a la misma unidad familiar:

ERPSo 47 = HEpOL 6370 (Osma BU): C(aius) Iulius Barbalrus Medutti/corum C(ai) f(ilius) / h(ic) s(itus) e(st) / Aemilia Acca / Medutticolrum Barbari / mater / h(ic) s(ita) e(st) / C(aius) Iulius Labeo / Crastunonis f(ilius) / Medutti(cor)um / h(ic) s(itus) e(st)

También nos encontramos con que una madre y una hija (suegra y mujer del dedicante, respectivamente) pertenecen a la misma unidad ${ }^{15}$ :

HEp.4.198 = HEpOL 6801 (Lara de los Infantes BU):

a) Ambatae [D]essic[a]e an (norum) LV Titus Va[---] soc[e] rae

b) [A]rceae Dessicae Pat [er]nif(iliae) an(norum) X[---] Titus uxso[ri]

Así pues, sistematizando todos estos datos podemos realizar las siguientes constataciones:

- marido y mujer suelen pertenecer a unidades diferentes, pero pueden pertenecer a la misma;

—el padre y sus hijos varones siempre pertenecen a la misma unidad;

— los hermanos varones siempre pertenecen a la misma unidad;

- las hermanas también pueden pertenecer a la misma unidad;

— padre e hija pueden pertenecer a la misma unidad o a unidades diferentes;

- madre e hijo pueden pertenecer a la misma unidad o a unidades diferentes;

- madre e hija pueden pertenecen a la misma unidad.

15 El valor de Dessica como nombre familiar heredado ya es señalado por Vallejo 2010, 634, así como por Navarro, Gorrochategui y Vallejo 2011, 116 y n. 116 . 
Si bien los datos no son demasiado abundantes, la conclusión a la que permiten llegar, como señaló M. ${ }^{a}$ Cruz González 1986, 101-110, es la de que los matrimonios debían ser exogámicos, es decir, entre miembros pertenecientes a unidades familiares diferentes. De la información que podemos obtener se desprende que la mujer debía pasar a pertenecer a la unidad del marido, aunque se conserva la memoria de su procedencia exógena, por lo que podía indicarse la unidad originaria a la que pertenecía antes de su matrimonio. En cambio, los hijos se encuadran por principio en la misma unidad familiar del padre, de forma que los varones pertenecerían a ella durante toda la vida, mientras que las mujeres quizá pudieran cambiar al casarse, de ahí que podamos encontrarnos con hijas que no pertenecen a la misma unidad que sus padres o con hijos que no pertenecen a la misma unidad que sus madres.

Según señalaran De Hoz 1986, 95-98, por varios motivos, y González 1986, 105, en función de los términos de parentesco que se encuentran en las inscripciones en las que aparecen estas unidades familiares, estas debían estar constituidas por un número bastante reducido de individuos, conclusión a la que también llega Salinas 2001, 171, quien, además, plantea la hipótesis de que estas entidades no existieran de forma general entre las comunidades indígenas, sino que estuvieran restringidas a miembros de las élites ${ }^{16}$. Para González el hecho de que los nombres de las unidades se relacionen con nombres personales frecuentes en la zona o, incluso, que aparezcan dentro de la misma inscripción, como los casos que tuvimos ocasión de ver en $\$ 1$, vendría a corroborar tal deducción ${ }^{17}$.

Creo que a este respecto es importante recordar aquí la información que se puede obtener de la inscripción de Cabezón de Liébana que vimos en la primera parte y en la que se mencionaba a un Ambatus Pentonieicus:

ERCan 102 = HEpOL 1072 (Cabezón de Liébana S): Mon(umentum) Ambati / Pentouieici Amblatiq(um) Pentoui f(ili) an(norum) LX/ hoc mon(umentum) p(osuerunt) Ambaltus et Doiderus f(ilii) / sui

Este dato, que, a pesar de ser tan interesante, había sido pasado por alto por la investigación, vendría a corroborar la conclusión de M. ${ }^{a}$ C. González 1986, 105 de que el nombre de la unidad familiar se formaba a partir del de un antepasado no demasiado lejano en el tiempo ni en grado de parentesco.

Desde esta perspectiva, podemos replantearnos ahora la función del nombre Vemenicus que veíamos en $\$ 2.4$ :

\section{ERLara $49=$ HEpOL 25717: Caeniues / Vemenicus / Beluicon [ - ] / SIS T R D HIC}

Quizá dicho nombre podría tener una función similar a la del Pentouieicus que hemos visto en la inscripción de Cabezón de Liébana, aunque los problemas de lectura nos dejan en este otro caso con más inseguridades. Señalemos, no obstante, que el nombre Vemenus, que es el que ha servido de base para la derivación de Vemenicus, es un hápax atestiguado únicamente en otra inscripción de Lara de los Infantes (ERLara $55=$ HEPOL 25722) ${ }^{18}$.

16 Véase también Salinas y Palao 2003, 405.

17 De hecho, Salinas 2001, 171 y Salinas y Palao 2003, 405, a propósito del área vetona, se planteaban, muy adecuadamente, si en vez de ante "organizaciones sociales», como se había asumido frecuentemente en la bibliografía, no nos encontramos más bien ante algún tipo de estructura familiar que existía en algunos sitios sí y en otros no, en función de tradiciones locales y otros factores.

18 Vallejo 2010, 637, incluye Vemenicus en su listado de nomina gentilicia con sufijo -icus añadido a una base indígena. 
En el mismo sentido, otro caso muy interesante es el de Medutica. Al igual que los anteriores, se documenta en una inscripción en la que aparece también la mención de la unidad familiar en genitivo plural:

AE 1916.73 = HEpOL 97 (Riba de Saelices GU): Arganto / Medutica / Melmanilq(um) a(nnorum) L(ucii) uxsolr h(ic) s(itus) e(st)

En la provincia de Soria, en cambio, tenemos atestiguado varias veces el genitivo plural de la unidad familiar correspondiente:

CIL II $2823=$ HEpOL 8649 (San Esteban de Gormaz SO): Nice $\cdot$ Atililae $\cdot$ Meduttiq(um) I anc(illa) $\cdot$ an (norum) $\cdot X X / h$ (ic) s(ita) e(st)

ERPSo 47 = HEpOL 6370 (Osma BU): C(aius) Iulius Barbalrus Meduttilcorum C(ai) f(ilius) / h(ic) s(itus) e(st) / Aemilia Acca / Medutticolrum Barbari / mater / h(ic) s(ita) e(st) / C(aius) Iulius Labeo / Crastunonis f(ilius) / Medutti(cor)um / h(ic) s(itus) e(st) Auia

HEp.7.257 = HEpOL 16475 (Coruña del Conde SO): Rustice / Meduttiq(um) / Aionis f(iliae) /

Naturalmente, no estoy proponiendo que necesariamente nos encontremos en todos los casos ante individuos de la misma estructura familiar, pero sí podría ser que en la inscripción de Riba de Saelices tengamos el precedente de lo que podría acabar siendo una unidad familiar de los $\mathrm{Me}$ duttici.

En todo caso, la inscripción de Cabezón de Liébana que hemos analizado parece apuntar a que esos «cognomina gentilicios", en la expresión de M. a Lourdes Albertos, o "cognomina hereditarios», en la de M. ${ }^{a}$ Cruz González, podían indicar la referencia a un antepasado no demasiado lejano que quizá en un par de generaciones podía llegar a proporcionar, a su vez, el nombre base para la designación de la unidad familiar o de una nueva rama. En cuanto al análisis de las relaciones de parentesco expresadas explícita o implícitamente en las inscripciones, viene a reforzar la idea ya defendida en estudios anteriores de que estas unidades familiares no eran grandes clanes, sino estructuras más reducidas y que quizá en varias generaciones podían fragmentarse y dar lugar, a su vez, a otras nuevas unidades con denominaciones diferentes a partir de antepasados de la familia no muy lejanos en el tiempo. De hecho, en los trabajos mencionados, Salinas ${ }^{19}$ se planteaba que estos grupos debían durar poco en el tiempo, quizá no más allá de tres o cuatro generaciones, lo cual cuadra bien con los datos a los que acabamos de hacer referencia.

\section{Conclusiones}

En este trabajo hemos partido del estudio de la relación entre los nombres de las unidades familiares indígenas expresados mediante genitivos de plural y los nombres indígenas (cognomina, nombres integrados en fórmulas con duo nomina y nombres únicos) que también pueden referirse a esas unidades familiares. Para la realización del trabajo hemos llevado a cabo una revisión exhaustiva de los materiales y, para proceder metodológicamente con cautela, hemos partido de aquellos

19 Salinas 2001, 171, y Salinas y Palao 2003, 403. 
casos en los que existe una coincidencia formal total entre los nombres de las unidades familiares en genitivo plural y los nombres indígenas en -icus que, siguiendo estudios anteriores, se había considerado que podían indicar la pertenencia a tales unidades familiares.

Hemos procedido a una revisión de la información que nos permite ofrecer argumentos adicionales a la mera coincidencia formal para apoyar dicha interpretación, como la constatación de que, al igual que sucede con los genitivos de plural, también se documentan en la misma inscripción nombres derivados en -icus y el nombre personal que les sirve de base y, sobre todo, la distribución geográfica. A este respecto hemos proporcionado una información exhaustiva sobre los nombres de unidades familiares que se documentan en más de una localidad y, basándonos en el estudio de la información explícita que proporcionan algunas inscripciones y en la propia dispersión de los nombres de las unidades, hemos proporcionado datos que creemos que apoyan la hipótesis formulada por Gómez Pantoja 1996 sobre la relevancia de la aparición del mismo nombre de unidad familiar en lugares distintos.

Tras analizar algunos ejemplos particulares de nombres posiblemente referidos a unidades familiares y los problemas que plantean, nos hemos ocupado de la relación entre los nombres de unidades familiares y nombres de divinidades en el occidente de la península Ibérica, aportando los datos de la documentación reciente a este respecto (las dos aras de Alcains, Castelo Branco, publicadas por Assunçāo, D’Encarnaçāo y Guerra 2009), en la que dicha relación resulta explícita, lo que necesariamente lleva a un replanteamiento de esta cuestión, a pesar de que ya había sido tratada en la bibliografía anterior.

Una reconsideración de las fórmulas onomásticas de Amparamus, individuo mencionado en las dos caras de la tésera de hospitalidad de Herrera de Pisuerga, nos ha permitido realizar varias constataciones, que pueden extenderse al resto de la documentación epigráfica y llevar a una reconsideración de diferentes elementos:

a) las dos fórmulas confirman que, incluso para un mismo individuo, la expresión de la pertenencia a una unidad familiar podía realizarse tanto mediante un genitivo plural como mediante una formación adjetival derivada, tal y como venía siendo defendido en la bibliografía al uso;

b) la fórmula Amparamus Nemaiecanus asegura que para la expresión de la pertenencia a una unidad familiar era posible la utilización de formaciones adjetivales derivadas diferentes de las en -ko-, que son las que habitualmente se contemplaban en la investigación, lo cual abre la posibilidad de considerar más nombres indígenas en fórmulas onomásticas como indicativos de la pertenencia a una unidad familiar, aunque esto exigirá siempre una gran cautela metodológica;

c) la comparación de gen. Nemaioq (um) frente al derivado Nemaiecanus permite constatar que pueden existir pequeñas variaciones morfológicas en la expresión del nombre de una misma unidad familiar, lo cual, como hemos planteado en $\$ 2.6$, abre la puerta a pensar que podemos estar ante la misma unidad familiar incluso en casos en los que no tenemos una coincidencia en los sufijos de derivación empleados, aunque también en este aspecto habrá que ser cautos metodológicamente y, en la medida de lo posible, intentar buscar argumentos adicionales que apoyen dicha identificación.

También hemos llevado a cabo una revisión exhaustiva de todas las inscripciones, tanto latinas como celtibéricas, que mencionan unidades familiares indígenas y que aportan, explícita o implícitamente, información sobre relaciones de parentesco, revisión de conjunto que no se había vuelto a realizar desde la monografía de M. ${ }^{a}$ Cruz González del año 1986. Esto nos ha permitido incorpo- 
rar a la discusión, además de datos puntuales procedentes de diferentes inscripciones, toda la rica información que a este respecto ofrece el tercer bronce de Botorrita. El análisis de los términos de parentesco celtibéricos atestiguados en este documento, en combinación con la estructura de las referencias a unidades familiares en genitivo plural que en él aparecen, nos ha permitido fundamentar la interpretación de la palabra launi como "esposa», cuyo uso en relación con la unidad familiar del marido es coherente con la información que se había podido obtener a partir de las inscripciones latinas, así como ofrecer interpretaciones para las secuencias en las que se mencionan los términos para «hijo»e «hija».

Esa revisión de las relaciones de parentesco en relación con los nombres de unidades familiares nos ha permitido corroborar la idea de que tales grupos familiares no debían ser grandes clanes o tribus, sino grupos familiares más reducidos que no debían duran más allá de unas pocas generaciones. Pero, además de este hecho, bien señalado en la bibliografía anterior, poner en relación tal constatación con la coexistencia dentro de una misma inscripción de nombres gentilicios en -cus y genitivos de plural referidos a unidades familiares indígenas (y también la existencia de unos pocos casos de dobles genitivos) nos ha posibilitado rastrear en la documentación epigráfica el proceso por el cual podía ir surgiendo, dentro de una unidad previamente existente, el germen de lo que acabaría siendo una nueva unidad familiar, con una denominación diferente que llegaría a sustituir a la anterior. Sabíamos previamente que, cuando tenemos documentación suficiente, los nombres de unidades familiares se relacionan de forma habitual con nombres personales de la misma localidad o área geográfica, pero una consideración más detallada de la coexistencia de nombres en -cus y genitivos de plural en casos afortunados como el de la inscripción de Cabezón de Liébana, a la que hemos hecho referencia tanto en $\$ 2.4$ como al final de $\$ 3$, nos ha permitido realizar algunos planteamientos adicionales sobre el surgimiento de nuevas unidades familiares con nuevos nombres.

La investigación sobre las unidades familiares en la Hispania antigua es un tema complejo y que tiene aún muchos interrogantes planteados. Confiamos en que con este trabajo hayamos contribuido, al menos, a abrir algunas posibilidades adicionales de indagación.

\section{BibLiOgRAFÍA}

Assunçấo, A., J. D’EnCarnação y A. Guerra, 2009, «Duas aras votivas romanas em Alcains», Revista Portuguesa de Arqueología 12.2, 177-189.

Barbas, R., E. Gamo y H. Gimeno, 2011, «Nuevos epígrafes latinos en el Alto Tajo: Abánades, Ocentejo y Zaorejas", Veleia 28, 161-173.

Beltrán, F., J. de Hoz y J. Untermann, 1996, El tercer bronce de Botorrita (Contrebia Belaisca), Zaragoza: Gobierno de Aragón.

Gómez Pantoja, J., 1996, «Gentilidad y origen», en: F. Villar y J. d'Encarnaçáo (eds.), La Hispania prerromana (Actas del VI Coloquio sobre Lenguas y Culturas Prerromanas de la Peninsula Ibérica), Salamanca: Universidad de Salamanca, 77-100.

González Rodríguez, M. ${ }^{a}$ C., 1986, Las unidades organizativas indígenas del área indoeuropea de Hispania, [Anejos de Veleia Series Maior n. ${ }^{\circ}$ ], Vitoria-Gasteiz: Universidad del País Vasco.

González Rodríguez, M. ${ }^{a}$ C. y M. Ramírez SÁnchez, 2011, "Unidades organizativas indígenas del área indoeuropea de Hispania III: addenda», Veleia 28, 253-267.

De Hoz, J., 1986, "La epigrafía celtibérica», en Reunión sobre epigrafía hispánica de época romano-republicana, Zaragoza: Institución Fernando el Católico, 43-102.

Jordán Cólera, C., 2004, Celtibérico, Monografías de Filología Griega 16, Zaragoza: Ediciones del Departamento de Ciencias de la Antigüedad-Universidad de Zaragoza. 
MLH IV = Untermann, J., 1997, Monumenta Linguarum Hispanicarum, vol. IV Die tartessischen, keltiberischen und lusitanischen Inschriften, Wiesbaden: Dr. Ludwig Reichert.

Navarro, M., J. Gorrochategui y J.M. ${ }^{a}$ Vallejo, 2011, "L'onomastique des Celtibères: de la dénomination celtibère à la dénomination romaine», en: M. Dondin-Payre (dir.), Les noms de personnes dans l'Empire romain. Transformations, adaptations, évolutions, Bordeaux : Ausonius, 89-174.

Prósper, B.M.a, 2007, «Varia Celtica Epigraphica. 1) Botorrita K.1.4. Nueva lectura e interpretación. 2) Nuevas organizaciones suprafamiliares del Occidente peninsular. 3) Tres divinidades de la Hispania celta: Aeiodaicino, Aiioragato, Boiogenae», Palaeohispanica 7, 161-174.

Ramírez SÁnchez, M. 1999, Epigrafía y organización social en la región celtibérica: los grupos de parentesco (Tesis doctoral), Las Palmas de Gran Canaria.

Salinas de FríAs, M., 2001, Los vettones. Indigenismo y romanización en el Occidente de la Meseta, Salamanca: Universidad de Salamanca.

Salinas de Frías, M. y J.J. Palao Vicente, 2003, «Eje 2. Estructuras familiares en el medio indígena», en: Grupo Mérida, Atlas antroponimico de la Lusitania romana, Mérida-Bordeaux: Fundación de Estudios Romanos-Ausonius Éditions, 401-406.

Vallejo, J.M. a 2010, "Los celtas y la onomástica. El caso hispano», en: Serta Palaeohispanica in Honorem Javier de Hoz (= Palaeohispanica 10), Zaragoza: Institución «Fernando el Católico», 629-647. 